\title{
Emergency management of atrial fibrillation
}

\author{
A Wakai, J O O'Neill
}

Postgrad Med J 2003;79:313-319

Atrial fibrillation is the most common cardiac arrhythmia managed by emergency and acute general physicians. There is increasing evidence that selected patients with acute atrial fibrillation can be safely managed in the emergency department without the need for hospital admission. Meanwhile, there is significant variation in the current emergency management of acute atrial fibrillation. This review discusses evidence based emergency management of atrial fibrillation. The principles of emergency management of acute atrial fibrillation and the subset of patients who may not need hospital admission are reviewed. Finally, the need for evidence based guidelines before emergency department based clinical pathways for the management of acute atrial fibrillation becomes routine clinical practice is highlighted.

See end of article for authors' affiliations

Correspondence to: Mr A Wakai, Department of Emergency Medicine, Beaumont Hospital, Beaumont Road, Dublin 9, Republic of Ireland; wakai@indigo.ie

Submitted

20 December 2002

Accepted

18 February 2003
A rial fibrillation is a supraventricular tachyarrhythmia characterised electrocardiographically by replacement of consistent $P$ waves by rapid, irregular, fibrillatory waves that vary in size, shape, and timing. It is associated with an irregular, frequently rapid, ventricular response when atrioventricular node conduction is intact.

Atrial fibrillation affects up to $4 \%$ of those over 60 years of age $\mathrm{e}^{1}$ and may be an independent risk factor for death. After adjustment for known risk factors, the relative risk of mortality is 1.5 for men and 1.9 for women. ${ }^{2}$ Atrial fibrillation is rare in the first two decades of life, but when it does occur is usually associated with congenital heart disease or an accessory pathway. ${ }^{3}$

The mechanism of initiation of atrial fibrillation is likely to be multifactorial and might be atrial vulnerability, contraction-excitation feedback, or a combination of these factors. ${ }^{4}$ Atrial fibrillation is frequently precipitated by underlying cardiac (hypertensive heart disease, heart failure, acute myocardial infarction, pericarditis, myocarditis, cardiac surgery) or non-cardiac disease (hyperthyroidism, pulmonary embolism, pneumonia, chronic obstructive pulmonary disease). Probably through atrial remodelling (frequently inflammation or fibrosis), conditions which precipitate atrial fibrillation act as a substrate for further development of atrial fibrillation. ${ }^{5}$

Atrial fibrillation is the most common sustained cardiac arrhythmia, accounting for approximately $35 \%$ of hospital admissions for cardiac arrhythmias. ${ }^{67}$ Hence, it is the most comrelated to the tachycardia cycle length, intrinsic mon form of cardiac arrhythmia emergency physicians manage. ${ }^{8-12}$ Meanwhile, its prevalence is increasing along with the aging population in the Western world?

With the advent of clinical decision units, and as more prolonged periods of management become common in the emergency department, the need to admit all patients with acute atrial fibrillation has been questioned..$^{1013-15}$ There is increasing evidence that selected patients with acute atrial fibrillation can be safely managed in an emergency department observation unit or clinical decision unit without the need for hospital admission. ${ }^{89^{16-18}}$ In one study, a third of patients with new onset atrial fibrillation did not require hospital admission. ${ }^{10}$ Furthermore, an emergency department based clinical pathway for the management of acute atrial fibrillation is associated with substantial reduction in hospital length of stay and a favourable trend of cost savings. ${ }^{17} 18$ Meanwhile, a recent survey of accident and emergency consultants in the United Kingdom revealed a wide variation in management practices of atrial fibrillation, especially with regard to cardioversion and anticoagulation therapy. ${ }^{19}$ It highlighted the need for evidence based guidelines for acute atrial fibrillation management in the emergency department setting.

\section{CLASSIFICATION OF ATRIAL \\ FIBRILLATION}

Although there is no universal consensus on the nomenclature or classification of atrial fibrillation, a simple and clinically relevant classification has been proposed by the American College of Cardiology/American Heart Association/ European Society of Cardiology practice guidelines for the management of atrial fibrillation..$^{20}$ In this classification, paroxysmal atrial fibrillation is self terminating; persistent atrial fibrillation is not self terminating and requires treatment for termination; in permanent atrial fibrillation, sinus rhythm cannot be sustained after cardioversion of atrial fibrillation or the patient and physician have decided to allow atrial fibrillation to continue without further efforts to restore sinus rhythm. $^{20}$ New onset atrial fibrillation may present in the emergency department setting as paroxysmal or persistent atrial fibrillation (fig 1). In this setting, patients presenting with symptomatic acute atrial fibrillation may have new onset

Abbreviations: AFFIRM, Atrial Fibrillation Follow-up Investigation in Rhythm Management (trial); INR, international normalised ratio; RACE, Rate Control versus Electrical Cardioversion for Persistent Atrial Fibrillation (trial); TDP, torsades de pointes; TOE, transoesophageal echocardiography 


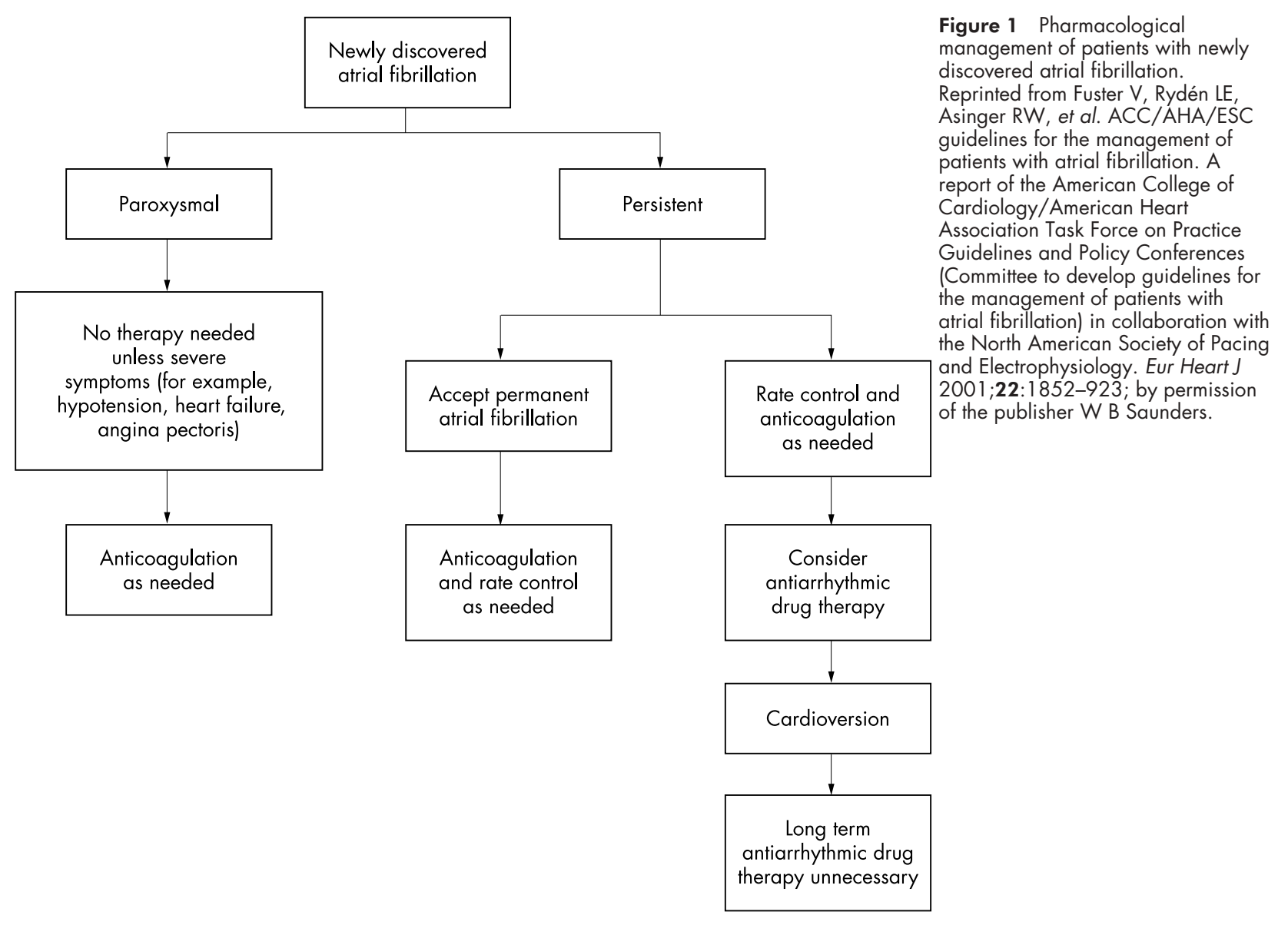

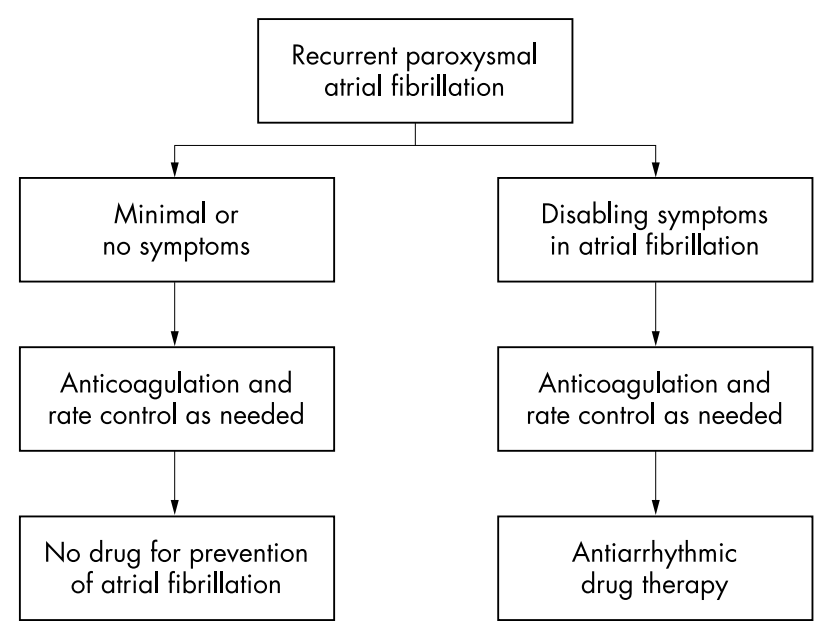

Figure 2 Pharmacological management of patients with recurrent paroxysmal atrial fibrillation. Reprinted from Fuster $\mathrm{V}$, Rydén LE, Asinger RW, et al. ACC/AHA/ESC guidelines for the management of patients with atrial fibrillation. A report of the American College of Cardiology/American Heart Association Task Force on Practice Guidelines and Policy Conferences (Committee to develop guidelines for the management of patients with atrial fibrillation) in collaboration with the North American Society of Pacing and Electrophysiology. Eur Heart J 2001 ;22:1852-923; by permission of the publisher W B Saunders.

atrial fibrillation (fig 1), recurrent paroxysmal (fig 2), or recurrent persistent atrial fibrillation (fig 3). Permanent atrial fibrillation is more likely to be an incidental finding in the emergency department setting.

The term "lone atrial fibrillation" describes atrial fibrillation in the absence of demonstrable underlying cardiac disease (coronary artery disease, heart failure, valvular heart disease, cardiomyopathy) or a history of hypertension. ${ }^{21}$ It occurs in approximately $3 \%-35 \%$ of patients with atrial fibrillation, depending on the population studied. ${ }^{22}{ }^{23}$ Lone atrial fibrillation may be due to pulmonary vein ectopic foci, ${ }^{24}$ proarrhythmic atrial fibrotic areas, ${ }^{25}$ increased susceptibility to autonomic neural stimuli to the heart, ${ }^{26}$ circulating autoantibodies against cardiac myosin heavy chains, ${ }^{27}$ or, very rarely, localised atrial myocarditis. ${ }^{28}$ In patients 60 years and younger, lone atrial fibrillation, although uncommon, has a very small risk of thromboembolism which does not justify warfarin therapy. ${ }^{22}$ In contrast, in patients 60 years or older with lone atrial fibrillation warfarin therapy is justified because of an increased risk of stroke and death. ${ }^{22} 29$

Patients with the Wolff-Parkinson-White syndrome presenting with acute atrial fibrillation represent a distinct entity, because special precautions are required in their emergency management. In this condition, there is prolonged atrial conduction and greater atrial vulnerability to development of atrial fibrillation. ${ }^{30}$ Rapid atrioventricular conduction over the accessory conduction pathway may precipitate fatal ventricular fibrillation. In the presence of Wolff-Parkinson-White syndrome, drugs that slow atrial fibrillation by blocking conduction through the atrioventricular node (digoxin, $\beta$-blockers, and calcium channel blockers) are contraindicated because they do not slow conduction over the accessory pathways and may therefore precipitate ventricular fibrillation.

\section{WHAT ARE THE PRINCIPLES OF EMERGENCY MANAGEMENT OF ATRIAL FIBRILLATION?}

In all patients presenting to the emergency department with acute atrial fibrillation, immediate provision of supplementary oxygen, establishment of an intravenous line, and continuous electrocardiographic monitoring is mandatory. In the emergency department assessment of atrial fibrillation (box 1), 


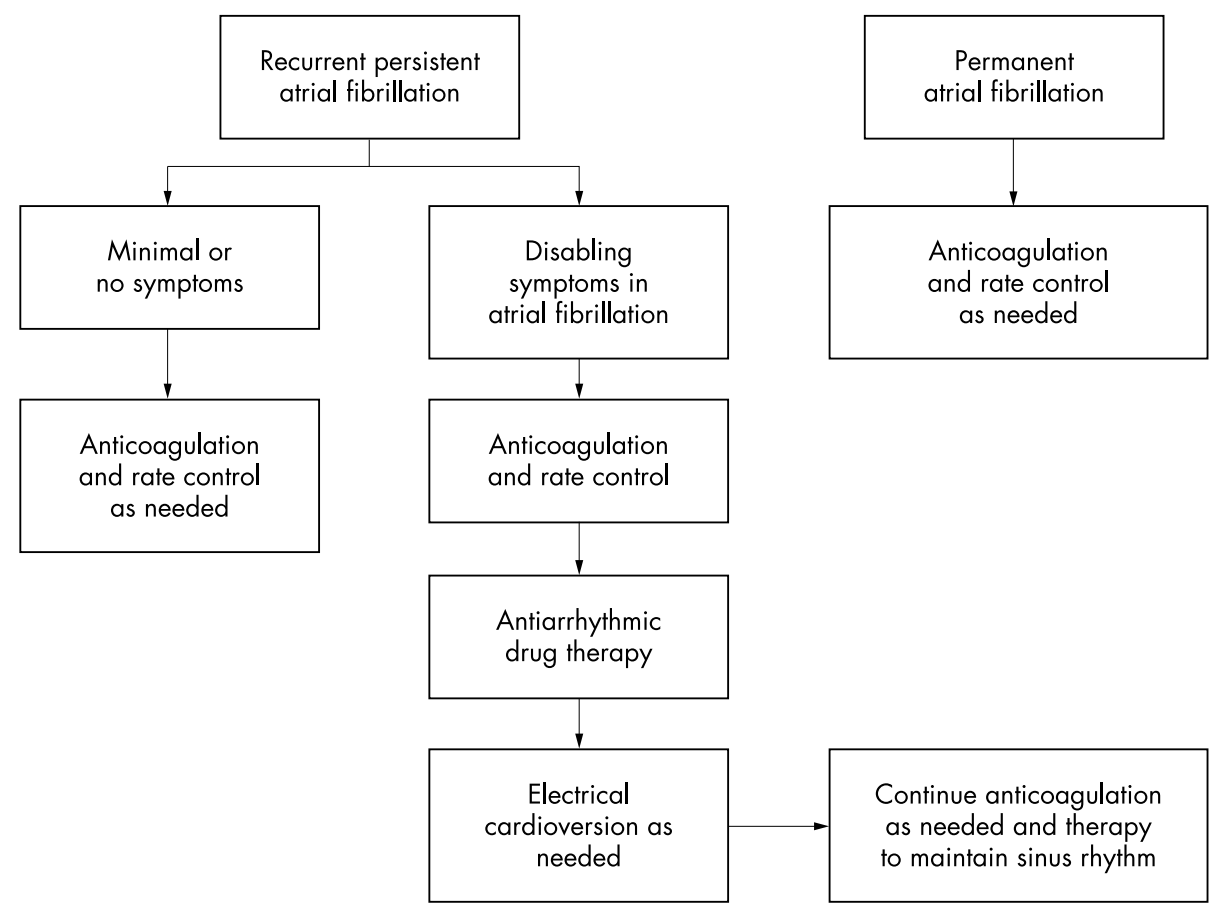

Figure 3 Pharmacological management of patients with recurrent persistent or permanent atrial fibrillation. Reprinted from Fuster $\mathrm{V}$, Rydén LE, Asinger RW, et al. ACC/AHA/ESC guidelines for the management of patients with atrial fibrillation. A report of the American College of Cardiology/American Heart Association Task Force on Practice Guidelines and Policy

Conferences (Committee to develop guidelines for the management of patients with atrial fibrillation) in collaboration with the North American Society of Pacing and Electrophysiology. Eur Heart $\mathrm{J}$ 2001;22:1852-923; by permission of the publisher W B Saunders.
Box 1: Emergency department assessment of atrial fibrillation

History

- Symptoms of atrial fibrillation (palpitations, dyspnoea, fatigue, polyuria, cognitive impairment).

- Alcohol consumption.

- Medications (proarrhythmic drugs).

Physical examination

- Signs of structural heart disease.

- Signs of alcohol withdrawal and hepatic failure.

- Signs of thyroid disease.

Investigations

- Electrocardiograhy.

- Chest radiography.

- Full blood count.

- International normalised ratio.

- Urea and electrolytes.

- Arterial blood gases.

- Cardiac enzymes.

- Liver function test.

- Blood glucose.

- Thyroid function test.

attention should also be paid to the need for alcohol withdrawal prophylaxis in patients with alcohol induced atrial fibrillation ("holiday heart syndrome") and alcohol induced liver disease.

The principles of emergency management of atrial fibrillation are prevention of thromboembolism, haemodynamic stabilisation, and symptom relief. These goals are achieved by a three part approach to management.

Firstly, to prevent the risk of embolic stroke associated with restoration of sinus rhythm (cardioversion), anticoagulation is required (figs 1-3). Although the risk of thromboembolism is low $(0.8 \%)$ if atrial fibrillation is of $<48$ hours' duration, ${ }^{3}$ emergency department patients should not be assumed to be free of left atrial thrombus because $15 \%$ of patients with acute atrial fibrillation (that is, $<3$ days) have evidence of atrial thrombi. ${ }^{32}$ Transoesophageal echocardiography (TOE) before cardioversion, to rule out occult left atrial appendage thrombi, is not presently available in the emergency department setting. Current guidelines state that in patients with atrial fibrillation undergoing cardioversion, anticoagulation therapy should be administered regardless of the method (chemical or electrical) used to restore sinus rhythm. ${ }^{20}$

Secondly, ventricular rate should be appropriately controlled. This is of primary importance in stable acute atrial fibrillation patients with rapid ventricular rate (figs 1-3). Allowing the persistence of atrial fibrillation but controlling the ventricular response has the advantage of avoiding the potential proarrhythmic side effects of antiarrhythmic drugs. The rate is generally considered controlled when the ventricular response ranges between 60 and 80 beats/min at rest. ${ }^{203}$

Thirdly, the need for, the proper timing of, and the appropriate method of cardioversion should be assessed. Cardioversion, theoretically, relieves symptoms, prevents thromboembolism, and prevents cardiomyopathy of tachycardia (tachycardiomyopathy). ${ }^{7034}$ It also improves cardiac output and exercise capacity, particularly in patients with heart failure. ${ }^{20}$

In symptomatic patients already taking warfarin, emergency management of acute atrial fibrillation depends on the international normalised ratio (INR). If it can be confirmed, from medical records, that the INR has been recently therapeutic (generally $>2.0$ ) for three or more weeks immediate cardioversion is indicated in haemodynamically unstable patients. However, since ventricular rate control can now be considered a primary management strategy in acute atrial fibrillation, ${ }^{356}$ it is more appropriate if the patient is haemodynamically stable and the INR is subtherapeutic or unknown.

\section{VENTRICULAR RATE CONTROL OR CARDIOVERSION IN ACUTE ATRIAL FIBRILLATION?}

Two different strategies exist for the emergency management of cardiac rhythm in patients with acute atrial fibrillation. The first entails allowing atrial fibrillation to persist while ventricular rate is controlled. The second is cardioversion and attempting to maintain sinus rhythm with antiarrhythmic drugs. Until recently ventricular rate control was intuitively considered somewhat inferior to cardioversion, because persistence of atrial fibrillation was thought to lead to 
continuation of symptoms. ${ }^{7}$ Furthermore, early cardioversion ( $<48$ hours after onset of atrial fibrillation) increases the rate of successful cardioversion, reduces the incidence of atrial fibrillation recurrence and avoids the need for long term anticoagulation. In addition, patients presenting to the emergency department with acute atrial fibrillation benefit from timely restoration of sinus rhythm because atrial fibrillation begets atrial fibrillation..$^{37}$ More importantly, restoration of sinus rhythm permits safe discharge from the emergency department without the need for hospital admission. ${ }^{8}{ }^{16-18}$

Nevertheless, the decision to cardiovert a patient with acute atrial fibrillation is not always simple because there is no solid evidence that cardioversion followed by prolonged maintenance of sinus rhythm is beneficial. ${ }^{20}$ Moreover, cardioversion exposes the patient to the risks associated with anticoagulation while in the emergency department. It also causes transient atrial dysfunction ("stunning"). ${ }^{38}$ In this regard, the duration of atrial fibrillation is an important consideration before cardioversion. Two days ( 48 hours) of acute atrial fibrillation is considered the timepoint beyond which left atrial appendage thrombi may form, and cardioversion induced atrial stunning is likely to occur. ${ }^{38}$ Thrombus formed during the period of stunning may be expelled after the return of atrial mechanical function. This may explain the clustering of thromboembolic events in the first 10 days after cardioversion ${ }^{39}$

Recent data from two large clinical trials, AFFIRM (Atrial Fibrillation Follow-up Investigation in Rhythm Management) and RACE (Rate Control versus Electrical Cardioversion for Persistent Atrial Fibrillation), validate ventricular rate control as a primary strategy for the management of acute atrial fibrillation. ${ }^{35} 36$ AFFIRM randomised patients to pharmacological treatment either to restore atrial rhythm or to control ventricular heart rate, whereas RACE compared pharmacological treatment to control heart rate with electrocardioversion of rhythm. Data from these trials indicate that rate control is cheaper and comparable to maintenance of sinus rhythm with regard to mortality, in those patients who can tolerate atrial fibrillation. ${ }^{35}{ }^{36}$ Moreover, in the AFFIRM trial, the survival curves did appear to separate at around 1.5-2 years in favour of the rate control group. Death and disabling stroke, hospitalisations, or new arrhythmias all tended to be less common in the rate control group..$^{35}$ In the RACE trial, patients in the rhythm control group experienced more thromboembolic complications, more heart failure, and more adverse effects. $^{36}$

\section{WHICH METHOD OF EMERGENCY CARDIOVERSION (ELECTRICAL OR CHEMICAL) IS OPTIMAL?}

Cardioversion should be attempted only after adequate anticoagulation. However, when acute atrial fibrillation produces haemodynamic instability in the form of angina pectoris, myocardial infarction, shock or pulmonary oedema, immediate cardioversion should not be delayed to achieve therapeutic anticoagulation. In this context, intravenous heparin or low molecular weight heparin should be initiated before cardioversion..$^{20}$

Cardioversion is achieved electrically (synchronous direct current countershock) or chemically (oral or intravenous antiarrhythmic drugs). The development of new antiarrhythmic drugs has increased the popularity of chemical (pharmacological) cardioversion, but some disadvantages persist, particularly the proarrhythmic effect of many of these drugs. Meanwhile, spontaneous conversion to sinus rhythm within 24 hours after the onset of atrial fibrillation is common, occurring in up to two thirds of patients. ${ }^{40}$ Once the duration of atrial fibrillation exceeds 24 hours, the likelihood of conversion decreases. After one week of persistent atrial fibrillation, spontaneous conversion is rare..$^{41}$
The choice of chemical or electrical cardioversion depends on the patient. For example, in patients with hypertension electrical cardioversion may be thrombogenic. ${ }^{36}$ Thus, in these patients, chemical cardioversion may be the method of choice for restoration of sinus rhythm. Available facilities and expertise (physician experience) in the emergency department also determines which method of cardioversion can be used. For example, it is desirable to have facilities with emergency external cardiac pacing available whenever electrical cardioversion is attempted because sinus asystole may occur after cardioversion.

Compared with electrical cardioversion, chemical cardioversion is simpler but less efficacious, being successful in approximately $50 \%$ of emergency department patients. ${ }^{16}$ However, no sedation or anaesthesia backup is required for successful chemical conversion. Emergency department electrical cardioversion is successful in approximately $80 \%$ to $89 \%$ of cases ${ }^{16}{ }^{17}$ but requires intravenous sedation. In this regard, short acting intravenous anaesthetic agents producing conscious sedation are preferable because rapid recovery postcardioversion may avoid hospital admission. ${ }^{42}$ Patients who undergo a failed attempt at chemical cardioversion can subsequently undergo successful electrical cardioversion. ${ }^{16}{ }^{17}$

Immediate electrical cardioversion is recommended in patients with acute atrial fibrillation and a rapid ventricular response associated with acute myocardial infarction, symptomatic hypertension, angina, or heart failure that does not respond promptly to pharmacological measures. ${ }^{20}$

\section{WHAT IS THE BEST PHARMACOLOGICAL AGENT FOR EMERGENCY VENTRICULAR RATE CONTROL?}

Ventricular rate control is achieved by depression of atrioventricular nodal conduction. Agents which may be administered to achieve emergency rate control include $\beta$-blockers (metoprolol, propranolol, esmolol), calcium channel blockers (verapamil, diltiazem), digoxin, or amiodarone..$^{20}$

These drugs depress atrioventricular nodal conduction by a variety of different mechanisms. ${ }^{43}$ Calcium channel blockers such as verapamil and diltiazem target the L-type calcium channel. Beta-blockers prolong atrioventricular refractoriness by targeting the $\beta$-adrenergic receptors. The predominant effect of digoxin on the atrioventricular node is mediated by enhanced vagal tone. ${ }^{44}$ Meanwhile, amiodarone has both sympatholytic and calcium antagonistic properties. ${ }^{20}$

The selection of a $\beta$-blocker or calcium channel blocker should be based on the physician's experience and the patient's clinical condition. For example, a $\beta$-blocker is preferable in patients with acute myocardial infarction or ischaemic heart disease but contraindicated in asthmatics. Although a direct comparison of rate control effect using different $\beta$-blockers has not been performed, theoretically, esmolol (an ultrashort acting $\beta$-blocker administered intravenously) may be the best $\beta$-blocker in the emergency department setting. It has the advantage in the emergency department setting of easy titration, due to its short plasma half life (nine minutes). ${ }^{45}$ Its main drawback is that it is cumbersome to use because multiple doses and multiple infusion steps are required. ${ }^{47}$

Verapamil and diltiazem are the calcium channel blockers commonly used for rate control in acute atrial fibrillation. Intravenously, each drug is effective in the emergency setting, but the response is transient, and repeated doses or a continuous intravenous infusion may be required to maintain heart rate control. ${ }^{20}$ Calcium channel blockers, particularly verapamil, are contraindicated in patients with heart failure.

In acute atrial fibrillation patients with Wolff-ParkinsonWhite syndrome calcium channel blockers and $\beta$-blockers are contraindicated for rate control because anterograde conduction along the accessory pathway is facilitated when these agents are administered. This may result in markedly 
increased ventricular rate, hypotension, and degeneration into ventricular fibrillation. In haemodynamically stable patients with Wolff-Parkinson-White syndrome, type I (for example, quinidine, procainamide, disopyramide) or type III (for example, ibutilide or amiodarone) antiarrhythmic agents may be used. ${ }^{20}$ When acute atrial fibrillation is associated with haemodynamic compromise in Wolff-Parkinson-White patients, early direct current cardioversion should be performed. ${ }^{20}$

When conventional measures fail, amiodarone is a suitable alternative agent for ventricular rate control in patients with acute atrial fibrillation. ${ }^{20}{ }^{48}$ However, a combination of drugs, with careful dose titration, may be necessary to control the rate of ventricular response in patients with acute atrial fibrillation ${ }^{20}$

\section{WHAT IS THE BEST PHARMACOLOGICAL AGENT FOR EMERGENCY CARDIOVERSION?}

In acute atrial fibrillation, several drugs are effective in restoring sinus rhythm. These include ibutilide, ${ }^{49}$ propafenone, ${ }^{50}$ flecainide, ${ }^{51}$ and sotalol. ${ }^{52}$ These agents increase the length of the interval between paroxysms but often do not totally abolish the arrhythmia. The treatment of acute atrial fibrillation with antiarrhythmic drugs that also slow the heart rate (such as sotalol) may simply convert symptomatic episodes to asymptomatic ones."

The success rate of individual drugs is highly variable from different studies because of differences in patient selection and definitions of successful cardioversion. Nevertheless, existing data suggest ibutilide is probably the most effective agent for the chemical cardioversion of acute atrial fibrillation in the emergency department setting. ${ }^{94}{ }^{55}$ Ibutilide has a rapid action (within one hour after administration) and is safe in patients with Wolff-Parkinson-White syndrome. ${ }^{20}$ Its main drawback is induction of torsades de pointes (TDP), with an incidence of $3.6 \%$ to $8.3 \%{ }^{49}{ }^{56}$ Electrocardiographic monitoring is, therefore, mandatory when using intravenous ibutilide. Patients should be monitored for at least four hours after initiation of ibutilide therapy. ${ }^{20}$ In contrast to other antiarrhythmic drug induced TDP (which usually occurs after successful cardioversion), ibutilide induced TDP may occur before conversion to sinus rhythm. ${ }^{49}$ Because of its rapid action, ibutilide induced TDP is usually transient (one to two hours) and no intervention is required in haemodynamically stable patients. ${ }^{9}$

\section{WHAT IS THE ROLE OF DIGOXIN IN THE EMERGENCY MANAGEMENT OF ATRIAL FIBRILLATION?}

Although digitalis compounds have been used to treat atrial fibrillation for over two centuries, more recent studies have shown that in some patients with atrial fibrillation, digoxin has little or no effect in terminating the arrhythmia, and may occasionally be detrimental. It may aggravate paroxysmal atrial fibrillation by shortening the atrial muscle refractory period and increasing the rate of fibrillation..$^{57}$ In new onset atrial fibrillation, digoxin is not effective in restoring sinus rhythm. ${ }^{58}$ Although digoxin may effectively slow the ventricular rate, there is a delay of at least 60 minutes before onset of a therapeutic effect in most patients, and peak systemic levels do not develop for up to six hours. ${ }^{20}$ Controlled studies have demonstrated that the conversion rate to sinus rhythm by digoxin is no better than placebo, although the effect of digoxin in slowing the ventricular response during atrial fibrillation is unquestioned. ${ }^{59}$ When digoxin is used alone, there is a tendency for a relapse into unstable heart rates after initial rate control. ${ }^{60}{ }^{61}$ Despite these limitations, digoxin is still considered by many emergency physicians as a first line agent for emergency department rate control in acute atrial fibrillation. ${ }^{19}$ Furthermore, many emergency physicians would administer digoxin intravenously, ${ }^{19}$ although there is little pharmacological advantage over the oral route, except in patients in whom it cannot be administered enterally. ${ }^{60}$

Because the predominant effect of digoxin on the atrioventricular node is mediated by enhanced vagal tone, it is useful for controlling the resting ventricular rate in atrial fibrillation, but ineffective for rate control in conditions of high sympathetic drive, including pyrexia or severe heart failure. ${ }^{60}$ In these situations, calcium channel blockers and $\beta$-blockers are more effective for rapid rate control in the emergency department setting.

The advantage of digoxin is its mild positive inotropic effect. It is, therefore, recommended that digoxin should mainly be used as a second line agent or reserved for those with heart failure or left ventricular dysfunction. ${ }^{9206162}$

\section{IS EMERGENCY DEPARTMENT INITIATION OF ANTIARRHYTHMIC PROPHYLAXIS FEASIBLE?}

After successful cardioversion, it is necessary to start the patient on an antiarrhythmic drug to prevent atrial fibrillation recurrence, if the patient has no identifiable reversible causes (figs 1-3)..$^{20}$ Drugs of choice to prevent recurrent atrial fibrillation and maintain normal sinus rhythm include class I (for example, procainamide) or class III (for example, sotalol and amiodarone) antiarrhythmic agents. ${ }^{43}$ Intravenous procainamide infusion $(2-5 \mathrm{mg} / \mathrm{min})$ is usually effective in preventing immediate recurrence of atrial fibrillation in the emergency department setting.9 More recently, "pure" class III agents such as dofetilide, a highly selective potassium channel blocker, have also been shown to effectively prevent recurrence of atrial fibrillation after cardioversion. ${ }^{63}$

Whether oral antiarrhythmic drug treatment should be initiated on an inpatient or outpatient basis is an ongoing debate. The decision depends on the patient symptoms, the presence of structural heart disease, and the type of drug. For example, initiation of sotalol or quinidine requires hospital admission because of the risk of TDP with these drugs. ${ }^{9}$ Outpatient initiation of antiarrhythmic treatment is feasible for individuals who are asymptomatic, have normal QT intervals on an electrocardiogram, and no significant heart disease. ${ }^{3064}$ Patients started on amiodarone may be safe for discharge after emergency cardioversion because of its minimal depression of myocardial function and low proarrhythmia potential. Amiodarone is currently the only agent for which there are substantial data to support initiation of outpatient treatment for atrial fibrillation. ${ }^{65-6}$

\section{WHICH SUBSET OF ATRIAL FIBRILLATION PATIENTS NEED HOSPITAL ADMISSION?}

Currently, a common practice in patients with acute atrial fibrillation is admission for rate control, anticoagulation, exclusion of myocardial infarction and other causes of atrial fibrillation, and possible cardioversion to restore sinus rhythm. This strategy usually incorporates a TOE guided strategy with cardioversion on intravenous heparin (if the TOE is negative for atrial thrombi) and subsequent anticoagulation with warfarin. ${ }^{18}$ Alternatively, patients are admitted for rate control and anticoagulation with subsequent outpatient elective cardioversion after therapeutic warfarin anticoagulation for three consecutive weeks. ${ }^{18}$

Hospitalisation can be limited to highly symptomatic patients, those with structural heart disease, those who have had an embolic event or are at high risk of thromboembolism (such as patients with rheumatic mitral valve disease), and patients with failure of rate control in the emergency department. ${ }^{1621}$ Inpatient electrocardiographic monitoring may also be required for those patients who, although successfully cardioverted in the emergency department, are started on oral antiarrhythmic therapy with high proarrhythmia potential (for example, sotalol and quinidine). ${ }^{9}$ 
Patients with non-cardiac causes of atrial fibrillation may also require hospital admission for appropriate management For example, when acute atrial fibrillation is associated with acute exacerbation of chronic obstructive pulmonary disease, management of the underlying lung disease with correction of hypoxia and acid balance are of primary importance. ${ }^{20}$

\section{WHICH SUBSET OF ATRIAL FIBRILLATION PATIENTS CAN BE SAFELY DISCHARGED FROM THE EMERGENCY DEPARTMENT?}

Broadly speaking, two categories of patients may be safely discharged from the emergency department: those with no structural heart disease and those who have a controlled ventricular rate. Patients younger than 60 years old with lone atrial fibrillation have a benign prognosis ${ }^{21}$ and may also not need hospital admission. Hospital admission is not necessary solely to rule out myocardial infarction in patients with new onset atrial fibrillation, in the absence of angina, electrocardiographic evidence of myocardial ischaemia, or a recent infarction. ${ }^{21}{ }^{68}$ Ischaemic heart disease rarely presents as atrial fibrillation in the absence of other signs or symptoms, except in elderly or diabetic patients.

Patients with persistent atrial fibrillation whose rhythm is converted to normal sinus rhythm by emergency department cardioversion can be discharged home after initiation of an appropriate oral antiarrhythmic treatment to prevent recurrence (fig 1). ${ }^{17}$ In patients with acute atrial fibrillation, especially if symptoms related to arrhythmia are minimal, acceptance of rate controlled atrial fibrillation is an option (figs $1-3$ ). ${ }^{35}{ }^{36}$ In a recent study, $4 \%$ of patients with acute atrial fibrillation managed in the emergency department were discharged home in rate controlled atrial fibrillation with no complications on follow up. ${ }^{17}$ All patients discharged home should be closely monitored, and both the patient and the physician should be alert to possible adverse drug effects. ${ }^{20}$

\section{CONCLUSIONS}

Spontaneous conversion to sinus rhythm occurs within 24 hours after onset of acute atrial fibrillation in up to two thirds of patients. In haemodynamically stable patients, for the moment, the default position when faced with a patient with acute atrial fibrillation must be to manage them conservatively with rate control rather than attempt cardioversion. In haemodynamically unstable patients, immediate electrical cardioversion is indicated.

Not all patients with acute atrial fibrillation presenting to an emergency department need hospital admission. It is feasible to safely manage and discharge a subset of patients with acute atrial fibrillation in an emergency department observation unit or clinical decision unit. Management should be individualised to each patient depending on several factors including patient symptoms, known duration of atrial fibrillation, and the presence of structural heart disease. Evidence based guidelines are required for safe and uniform emergency department management of acute atrial fibrillation due to the current wide variation in management practices and unnecessary hospital admission of some patients with acute atrial fibrillation.

\section{CONTRIBUTORS}

Abel Wakai initiated the review, searched the emergency medicine literature for relevant papers, and prepared the first draft of the manuscript. James O O'Neill searched the cardiology literature for relevant papers and appraised the manuscript for conformity to cardiology practice. Abel Wakai and James $\mathrm{O} \mathrm{O}^{\prime}$ Neill produced the final manuscript. Abel Wakai acts as the guarantor for the paper.

\section{Authors' affiliations}

A Wakai, Department of Emergency Medicine, Beaumont Hospital, Dublin, Republic of Ireland
J O O'Neill, Department of Medicine, Our Lady's Hospital, Navan, Republic of Ireland

\section{REFERENCES}

1 Benjamin EJ, Levy D, Vaziri SM. Independent risk factors for atrial fibrillation in a population-based cohort: the Framingham Heart Study. JAMA 1994;271:840-4.

2 Benjamin EJ, Wolf PA, D'Agostino RB, et al. Impact of atrial fibrillation on the risk of death: the Framingham Heart Study. Circulation 1998:98:946-52.

3 Prystowsky EN, Benson DW, Fuster V, et al. Management of patients with atrial fibrillation: a statement for healthcare professionals from the subcommittee on electrocardiography and electrophysiology, American Heart Association. Circulation 1996;93:1262-77.

4 Prystowsky EN. Tachycardia-induced tachycardia: a mechanism of initiation of atrial fibrillation. In: DiMarco JP, Prystowsky EN, eds. Atrial arrhythmias: state of the art. Armonk, NY: Futura Publishing Co, 1995

arrhythmias: state of the art. Armonk, NY: Futura Publishing Co, 1995 .
5 Falk RH. Etiology and complications of atrial fibrillation: insights from pathology studies. Am J Cardiol 1998;82:10-17N.

6 Bialy D, Lehmann MH, Schumacher DN, et al. Hospitalisation of arrhythmias in the United States: importance of atrial fibrillation. J Am Coll Cardiol 1992:19:41A

7 Prystowsky EN, Katz A. Atrial fibrillation. In: Topol EJ, ed. Textbook of cardiovascular medicine. 2nd Ed. Philadelphia: Lippincott Williams \& Wilkins, 2002.

8 Connors S, Dorian P. Management of supraventricular tachycardia in the emergency department. Can J Cardiol 1997; 13:19A-24.

9 Li H, Easley A, Barrington W, et al. Evaluation and management of atrial fibrillation in the emergency department. Emerg Med Clin North Am 1998:16:389-403

10 Mulcahy B, Coates WC, Hennerman PL, et al. New-onset atrial fibrillation: when is admission medical justified? Acad Emerg Med 1996;3:1 14-19.

11 Feinberg WM, Blackshear JL, Laupacis A, et al. Prevalence, age distribution, and gender of patients with atrial fibrillation: analysis and implications. Arch Intern Med 1995;155:469-73.

12 The National Heart, Lung, and Blood Institute (NHLBI) Working Group on Atrial Fibrillation. Atrial fibrillation: current understandings and research imperatives. J Am Coll Cardiol 1993;22:1830-4.

13 Schreckengost VE, Zimetbaum PJ, Lemery R, et al. A prospective evaluation of the outpatient initiation of anti-arrhythmic drug therapy in patients with atrial fibrillation or flutter. Clin Cardiol 1997; suppl patients with
$1: 1-454$.

14 Hollander JE, Phibbs BP, Henneman PL, et al. Atrial fibrillation and hospital admission [letter]. Acad Emerg Med 1996;3:819-21.

15 Botto GL, Boninni W, Broffoni T, et al. Conversion of recent atrial fibrillation with a single loading dose of propafenone: is in-hospital admission absolutely necessary? PACE 1996:19:1939-43.

16 Michael JA, Steill IG, Agarwal S, et al. Cardioversion of paroxysmal atrial fibrillation in the emergency department. Ann Emerg Med 1999;33:379-87

17 Koenig BO, Ross MA, Jackson RE. An emergency department observation unit protocol for acute-onset atrial fibrillation is feasible. Ann Emerg Med 2002:39:374-81.

$18 \mathrm{Kim} \mathrm{MH}$, Morady F, Conlon B, et al. A prospective, randomized controlled trial of an emergency department-based atrial fibrillation treatment strategy with low-molecular-weight heparin. Ann Emerg Med 2002;40: 187-92

19 Williams E, Ansari M, Lip GYH. Managing atrial fibrillation in the accident and emergency department. QJ Med 2001;94:609-14

20 Fuster V, Rydén LE, Asinger RW, et al. ACC/AHA/ESC guidelines for the management of patients with atrial fibrillation. A report of the American College of Cardiology/American Heart Association Task Force on Practice Guidelines and Policy Conferences (Committee to develop guidelines for the management of patients with atrial fibrillation) in collaboration with the North American Society of Pacing and Electrophysiology. Eur Heart J 2001 22:1852-923.

21 Falk RH. Atrial fibrillation. N Engl J Med 2001;344:1076-8.

22 Kopecky SL, Gersh BJ, McGoon MD, et al. Lone atrial fibrillation in elderly persons: a marker for cardiovascular risk. Arch Intern Med 1999; 159:1118-22.

23 Prystowsky EN, Margiotti R, Fogel RI, et al. Atrial fibrillation with and without heart disease: clinical characteristics and proarrhythmia risk. Circulation 1996:94: I-191.

24 Haïssaguerre $M$, Jaïs $P$, Shah DC, et al. Spontaneous initiation of atrial fibrillation by ectopic beats originating in the pulmonary veins. N Engl J Med 1998;339:659-66

25 Bharati S, Lev M. Histology of the normal and diseased atrium. In: Falk $\mathrm{RH}$, Podrid PJ, eds. Atrial fibrillation: mechanisms and management. New York: Raven Press, 1992

26 Chen YJ, Chen SA, Tai CT, et al. Role of atrial electrophysiology and autonomic nervous system in patients with supraventricular tachycardia and paroxysmal atrial fibrillation. J Am Coll Cardiol 1998;32:732-8.

27 Maixent JM, Paganelli F, Scaglione J, et al. Antibodies against myosin in sera of patients with idiopathic paroxysmal atrial fibrillation. $J$ Cardiovasc Electrophysiol 1998:9:612-7.

28 Frustaci A, Chimenti C, Bellocci F, et al. Histological substrate of atrial biopsies in patients with lone atrial fibrillation. Circulation 1997;96: 1180-4 
29 Jouven X, Desnos M, Guerot C, et al. Idiopathic atrial fibrillation as a risk factor of mortality: the Paris Prospective Study I. Eur Heart J 1999:20:896-9

30 Hamada T, Hiraki T, lkeda $\mathrm{H}$, et al. Mechanisms for atrial fibrillation in patients with Wolff-Parkinson-White syndrome. J Cardiovasc Electrophysiol 2002;13:223-9.

31 Weigner MJ, Caulfield TA. Risk of clinical thromboembolism associated with conversion to sinus rhythm in patients with atrial fibrillation lasting less than 48 hours. Ann Intern Med 1997: 126:615-20.

32 Stoddard MF, Dawkins PR, Prince CR, et al. Left atrial appendage thrombus is not uncommon in patients with acute atrial fibrillation and a recent embolic event: a transesophageal echocardiographic study. J Am Coll Cardiol 1995;25:452-9.

33 Rawles JM. What is meant by a "controlled" ventricular rate in atrial fibrillation? Br Heart J 1990;63:157-61

34 Manning WJ, Silverman DI, Katz SE, et al. Impaired left atrial mechanical function after cardioversion: relation to the duration of atrial fibrillation. J Am Coll Cardiol 1994;23:1535-40.

35 The Atrial Fibrillation Follow-up Investigation of Rhythm Management (AFFIRM) Investigators. A comparison of rate control and rhythm control in patients with atrial fibrillation. N Engl J Med 2002:347:1825-33.

36 Van Gelder IC, Hagens VE, Bosker HA, et al. A comparison of rate control and rhythm control in patients with recurrent persistent atrial fibrillation. N Engl J Med 2002;347:1834-40.

37 Wijffels MCEF, Kirchhof CJHJ, Dorland R, et al. Atrial fibrillation begets atrial fibrillation: a study in awake chronically instrumented goats. Circulation 1995:92:1954-68.

38 Silverman DI, Manning WJ. Role of echocardiography in patients undergoing elective cardioversion of atrial fibrillation. Circulation 1998;98:479-86

39 Berger $M$, Schweitzer P. Timing of thromboembolic events after electrica cardioversion of atrial fibrillation or flutter: a retrospective analysis. Am J Cardiol 1998;82:1545-7, A8.

40 Danias PG, Caulfield TA, Weigner M, et al. Likelihood of spontaneous conversion of atrial fibrillation to sinus rhythm. J Am Coll Cardiol 1998:31:588-92

41 Reisinger J, Gatterer E, Heinze G, et al. Prospective comparison of flecainide versus sotalol for immediate cardioversion of atrial fibrillation. Am J Cardiol 1998;81:1450-4.

42 Lesser MF. Safety and efficacy of in-office cardioversion for treatment of supraventricular arrhythmias. Am J Cardiol 1990;66:1267-8.

43 Lévy S. Pharmacologic management of atrial fibrillation: current therapeutic strategies. Am Heart J 2001;141:S15-21.

44 Falk RH, Leavitt Jl. Digoxin for atrial fibrillation: a drug whose time has gone? Ann Intern Med 1991;114:573-85.

45 Elam K, Bolar-Softich KL. Dilemmas in the acute pharmacologic treatment of uncontrolled atrial fibrillation. Am J Emerg Med 1997:15:418-9.

46 Barbier GH, Shettigar UR, Appunn DO. Clinical rationale for the use of an ultra-short acting beta-blocker: esmolol. Int J Clin Pharmacol Ther 1995;33:212-8.

47 Pritchett EL. Management of atrial fibrillation. N Engl J Med 1992;326:1264-71

48 Clemo HF, Wood MA, Gilligan DM, et al. Intravenous amiodarone for acute heart rate control in the critically ill with atrial tachyarrhythmias. Am J Cardiol 1998:81:594-8.
49 Stambler BS, Wood MA, Ellenbogen KA, et al. Efficacy and safety of repeated intravenous doses of ibutilide for rapid conversion of atrial flutter or fibrillation. Circulation 1996:94:1613-21.

50 Cobbe SM, Rae AP, Poloniecki JD, et al. A randomized, placebo-controlled trial of propafenone in the prophylaxis of paroxysmal supraventricular tachycardia and paroxysmal atrial fibrillation. Circulation 1995;92:2550-7

51 Martinez-Marcos FJ, Garcia-Garmendia JL, Ortega-Carpio A, et al. Comparison of intravenous flecainide, propafenone, and amiodarone for conversion of acute atrial fibrillation to sinus rhythm. Am J Cardiol 2000:86:950-3.

52 Lee SH, Chen SA, Tai CT, et al. Comparisons of oral propafenone and sotalol as an initial treatment in patients with symptomatic paroxysmal atrial fibrillation. Am J Cardiol 1997;79:905-8.

53 Juul-Möller S, Edvardsson N, Rehnqvist-Ahlberg N. Sotalol versus quinidine for the maintenance of sinus rhythm after direct current conversion of atrial fibrillation. Circulation 1990:82:1932-9.

54 Volgman AS, Stambler BS, Kappagoda C, et al. Comparison of intravenous ibutilide versus procainamide for the rapid termination of atrial fibrillation or flutter [abstract]. PACE 1996;19:608.

55 Vos MA, Golitsin SR, Stangl K, et al. Superiority of ibutilide (a new class III agent) over DL-sotalol in converting atrial flutter and fibrillation. Heart 1998;79:568-73

56 Ellenbogen KA, Stambler BS, Wood M, et al. Efficacy of intravenous ibutilide for rapid termination of atrial fibrillation and atrial flutter: a dose-response study. J Am Coll Cardiol 1996;28:130-6.

57 Rawles JM, Metcalfe M. Jennings K. Time of occurrence, duration, and ventricular rate of paroxysmal atrial fibrillation: the effect of digoxin. The Cardiac Arrhythmia Suppression Trial. Br Heart J 1990;63:225-7.

58 Intravenous digoxin in acute atrial fibrillation. Results of a randomized, placebo- controlled multicentre trial in 239 patients. The Digitalis in Acute Atrial Fibrillation (DAAF) Trial Group. Eur Heart J 1997; 18:649-54

59 Jordaens $\mathrm{L}$, Troverbach J, Calle $\mathrm{P}$, et al. Conversion of atrial fibrillation to sinus rhythm and rate control by digoxin in comparison to placebo. Eur Heart J 1997; 18:643-8.

60 Li Saw Hee FL, Lip GYH. Digoxin revisited. Q J Med 1998;91:259-64.

61 Lip GYH. How would I manage a 60 -year old woman presenting with atrial fibrillation? Proc Roy Coll Phys Edinb 1999;29:301-6.

62 Jung F, DiMarco JP. Treatment strategies for atrial fibrillation. Am J Med 1998;104:272-83.

63 Castro A, Biaconi L, Santini M. New antiarrhythmic drugs for the treatment of atrial fibrillation. Pacing Clin Electrophysiol 2002:25:249-59.

64 Prystowsky EN. Management of atrial fibrillation: therapeutic options and clinical decisions. Am J Cardiol 2000;85:3-11D.

65 Gosselink M, Crijns HJG, Van Gelder I, et al. Low-dose amiodarone for maintenance of sinus rhythm after cardioversion of atrial fibrillation or flutter. JAMA 1992;267:3289-93.

66 Kockiadakis G, Igoumenidis N, Solomou M, et al. Efficacy of amiodarone for the termination of persistent atrial fibrillation. Am J Cardiol 1999;83:58-61

67 Kockiadakis G, Igoumenidis N, Parthenakis F, et al. Amiodarone versus propafenone for conversion of chronic atrial fibrillation: results of a randomized, controlled study. J Am Coll Cardiol 1999;33:966-71.

68 Zimetbaum PJ, Josephson ME, McDonald MJ et al. Incidence and predictors of myocardial infarction among patients with atrial fibrillation. J Am Coll Cardiol 2000;36:1223-7. 